\title{
A Reverse Phase Protein Array Based Phospho- antibody Characterization Approach and Its Applicability for Clinical Derived Tissue Specimens
}

\section{Nan Wang ( $\square$ nan.wang@fynnbio.com )}

Fynn Biotechnologies https://orcid.org/0000-0001-5801-7399

\section{Li Zhang}

Beijing Cancer Hospital

Qi Ying

Fynn Biotechnologies

\section{Zhentao Song}

Fynn Biotechnologies

Aiping Lu

Beijing Cancer Hospital

\section{Achim Treumann}

KBI Biopharma

\section{Zhaojian Liu}

Shandong University Cheeloo College of Medicine

\section{Tao Sun}

Shandong University Cheeloo College of Medicine

\section{Zhiyong Ding}

Fynn Biotechnologies

\section{Research}

Keywords: Reverse phase protein array, Signaling pathways, Phospho-antibody profiling, Fresh frozen tissue, formalin-fixed and paraffin-embedded tissue

Posted Date: August 19th, 2021

DOl: https://doi.org/10.21203/rs.3.rs-806139/v1

License: (c) (1) This work is licensed under a Creative Commons Attribution 4.0 International License.

Read Full License 


\section{Abstract}

\section{Background:}

Systematic quantification of phosphoprotein within cell signaling networks in solid tissues remains challenging and precise quantification in large scale samples has great potential for biomarker identification and validation.

\section{Methods:}

We developed a reverse phase protein array (RPPA) based phosphor-antibody characterization workflow by taking advantage of the lysis buffer compatible with alkaline phosphatase (AP) treatment and here termed it as a bottom-up antibody screening that differs from the conventional RPPA antibody validation procedure and applied it onto fresh frozen and formalin-fixed and paraffin-embedded tissue (FFPE) to test its applicability.

\section{Results:}

We tested the feasibility of this method by screening 106 phospho-antibodies with RPPA first followed by western blots on a panel of cell lines and demonstrated that AP treatment could serve as an independent factor that can be adopted for rapid RPPA phospho-antibody selection. We also performed studies on different clinical materials. For fresh frozen (FF) samples, pre-selected highly-specific antibodies showed a desirable data reproducibility and antibody specificity based on AP treatment indicating a potential for fresh tissue-based phospho-protein RPPA profiling. Of further clinical significance, using the same approach, based on two sets of FFPE samples from 63 melanoma and 40 lung cancer patients, we showed great interexperimental reproducibility and significant correlation with pathological markers MelanA for melanoma as well as a panel of lung cancer biomarkers for subtyping (EGFR, Napsin A, p63/p40, TTF1 and CK7) generating meaningful data that match clinical features.

\section{Conclusions:}

Our findings establish a highly efficient approach for phospho-antibody characterization by taking advantage of RPPA whereby the same methodology can be applied for tissue-based proteomics and phosphoproteomics in clinical assay development and application.

\section{Background}

Owing to its flexible and multiplexing capability as well as miniaturized and highly quantitative dot-blot nature, Reverse Phase Protein Arrays (RPPA) have been adopted in many laboratories worldwide. It serves as a powerful tool particularly for low abundant protein measurement and thus a great technique for quantitative proteomic analysis from trace amount of materials such as patient tissues (1). The focus of RPPA has primarily been its application within the oncology field wherein cell signaling dynamics and networking play a pivotal role and it is also the fact that the abundance of those signaling molecules 
such as post-translational modifications (PTMs) are too extreme to quantify. RPPA has been extensively integrated into various research contexts where its predominant application and advantage can be summarized into three-fold: 1) to elucidate underlying mechanisms with certain biological backgrounds from functional proteomics perspectives. These correlate with functions of specific genes or proteins, a particular drug or drug combinations either in vitro or in vivo from basic research to clinical translational studies. 2) to allow parallel multi-omics profiling incorporating genetics, transcriptomics and proteomics within the same set of tissue samples. This facilitates the discovery and validation of diagnostic prognostic biomarkers linking with accessible clinical data in combination with other omics findings and adding a new dimension of data to support drug development. 3) to serve as a foundation for clinical assay development for personalized cancer treatment. This requires thorough evaluation and validation of multiple drug targets and their activity status based on various tissue types and generation of clinically feasible pipeline that can be Clinical Laboratory Improvement Amendments (CLIA) cleared and serves as Laboratory Developed Tests (LDT). All abovementioned aspects highlight the usability of RPPA as an ideal technique for clinical tissue based proteomic profiling.

However, notwithstanding the effort made in fine-toning the tissue based RPPA application, several technical bottlenecks still exist. Validation of RPPA-applicable antibodies is a prerequisite for accurately capture target antigens present under the denatured condition. This relates to the composition of the lysis buffer as this may have significant impact on the tissue solubility, signal intensity, reproducibility and dynamic range as well as downstream processes such as blocking and detection methods that may have unpredictable impact on the quantification (2-4). It is generally accepted to perform RPPA antibody prescreening using western blots (WB) where the standard criteria are predominant or explainable bands appearing on the blots at correct molecular mass ranges and more importantly, a good correlation between RPPA and WB performed on the same set of samples is a major determinant for a validated antibody (5-9). In terms of phospho-antibody validation, more complicated strategies can be used such as perturbation with various stimuli or specific inhibitors for a particular protein of interest $(3,4)$. In addition, for tissue based RPPA profiling, separate antibody validation is recommended as certain discrepancy may exist between tissues and cell lines due to the heterogeneity population causing decreased specificity as well as preanalytical variables to affect phosphoprotein levels including cold ischemia, hypoxia, hydration/dehydration as well temperature fluctuation $(9,10)$. These altogether complicate the antibody validation under a specific research context and therefore a better phosphoantibody characterization strategy is in need. Alkaline phosphatase (AP) treatment of samples to serve as negative controls has been suggested using other techniques in a medium-to-low throughput manner, however its application as a high throughput phospho-antibody screening strategy using RPPA has merely been suggested and in theory this could serve as an ideal negative control to evaluate the performance of antibody directly on chip $(7,11)$. Nevertheless, apart from one study where researchers applied tyrosine phosphatase treatment on phospho-peptide to serve as a negative array control for antibody reference standard development (12), there is no systematic evidence yet to prove its applicability either in cell line or tissue based RPPA profiling. 
On the other hand, it is also generally accepted that formalin-fixed paraffin-embedding (FFPE) is a universal tissue preparation method for pathological processing and this also lies in the scope of RPPA application whereby researchers have made tremendous dedication on improving the preclinical and clinical applicability of the technology (13-18). Many groups have established methods for efficient protein extraction from FFPE samples for downstream RPPA profiling and most protocols use $1-2 \%$ SDS to allow solubilization of hydrophobic proteins such as transmembrane proteins and a heating step for crosslinking reversal as well as other pre-and post-steps including deparaffinization, rehydration, sonication to obtain optimal condition for full-length protein extraction $(13,19)$. Side by side comparison between RPPA and immunohistochemistry (IHC) were underway. Many researchers evaluated the quantitative capability of RPPA using several routine pathological markers for IHC and found that human epidermal growth factor receptor 2 (HER2) exhibited nearly 100\% concordance rate based on a SuperCurve signal intensity cutoff of 1600 in a cohort of 35 breast cancer FFPE samples but not for estrogen receptor (ERa) and progesterone receptor (PR) assayed simultaneously (19). Similar studies have also found good correlation with $2+/ 3+$ IHC level of HER2 expression with RPPA (18). Another group assessed the correlation between RPPA and IHC with two sets of FFPE samples. One set consisting of 19 breast cancer specimens for IHC HER2 score $0,1+, 2+, 3+$ were used to build an ordinal multinomial logistic model for prediction on a testing set of 27 specimens (19). The training set generated a spearman correction of $0.86(\mathrm{p}<0.05)$ and concordance rates of $\mathrm{k}=0.673$ and $\mathrm{k}=0.403$ on the training and validation sets respectively. Using a non-small cell lung cancer (NSCLC) set of 50 patients, in line with pathological features they found Napsin A and cytokeratin5 were differentially expression between adenocarcinoma (ADC) and squamous cell carcinoma (SCC) and by profiling with 150 protein expression using RPPA, they discovered significantly higher expression of PAK2 in SCC than ADC indicating a potential role in tumorigenesis (20). In spite of those contributions made to develop a potential FFPE compatible standard for future clinical use, evidence is still limited to very few markers being tested and therefore further works are in need to potentiate the clinical applicability of RPPA for FFPE based profiling as a companion diagnostic tool.

More importantly, another technical bottleneck exists since antibodies for FFPE sample based RPPA need to be validated independently due to discordance of antibody performance observed between FF and FFPE samples. One study demonstrated that there is around $40 \%$ concordance rate for 300 antibodies profiled on xenograft prepared FFPE samples compared to their matching FF (21). By profiling three panels of antibodies on three sample sets (cell lines, breast cancer tissues, renal cancer tissues) consisting of FFPE and counterpart FF, researchers observed varied correlation between these two forms of specimens with $45 \%$ for cell lines, $32 \%$ for breast cancer and $64 \%$ for renal cancer respectively ( $p$ value<0.05) (22). These altogether suggest that antibodies for RPPA application is context dependent and rigorous validation on antibodies across various FFPE tissue types is of great importance for potential clinical application and those potential antibody subsets may demonstrate clinical robustness and further work will be needed to fill this gap.

In this multi-purpose study, in order to provide further evidence for RPPA as a multi-plex clinical proteomic tool, we first developed a RPPA phospho-antibody validation workflow using an in-house developed lysis 
buffer that maintains certain levels of enzymatic activity of AP and thereby facilitates global phosphogroup removal from proteins and use them as negative controls for phospho-antibody validation for RPPA directly on-chip. We term this fast-screening procedure as a bottom-up method starting off from RPPA and then re-validating the pre-selected antibody specificity using WB. Secondly, we further compared this buffer with other extraction systems to demonstrate its feasibility and advantages on FF samples especially for phosphor marker profiling taking advantage of the AP treatment controls. Finally, we assessed the FFPE applicability broadly with two independent sets of samples consisting of 63 melanoma patients and 40 lung cancer patients for three different subtypes generating clinical interpretable data with a potential of parallel high-plex proteomic profiling.

\section{Methods}

Cell Lines and Tissue Sample Acquisition

For RPPA screening, eight cell lines (Huh7, HFC, MCF7, SEM, 293T, LNCaP, LX-7, U2OS) were obtained from various commercial vendors and their identities were confirmed using Short Tandem Repeat assays (STR). All the rest of cells used for western blotting were also STR confirmed (all cell line information is included in Supplemental S-1). Cells were routinely cultured in recommended medium with $5 \% \mathrm{CO} 2$ and $10 \%$ fetal calf serum (FCS). EGF, IGF and Insulin treatment for phosphor-antibody validation were obtained from ProSpec (CYT-217, CYT-216) and Signa (12643) respectively. Transiently transfected overexpression clones in 293T cells were conducted by VigeneBio (www.vigenebio.cn). Breast tumor tissue specimens (FF and FFPE, 10 $\mu \mathrm{m}$ in thickness) used for extraction optimization, buffer comparison and AP treatment were obtained from Department of Pathology in Beijing Cancer Hospital. 189 Melanoma patient FFPE slides derived from 63 patients and 120 lung cancer patient and normal FFPE tissues from total of 40 individuals ( $1 \mathrm{~cm}^{2}$ size, $10 \mu \mathrm{m}$ in thickness) were also obtained from Beijing Cancer Hospital upon internal review board approval with written consent from patients for research purpose use. Tissue samples were all confirmed with neoplastic cellular content of over $50 \%$ as passing criteria.

Protein Extraction and Alkaline Phosphatase Treatment for Phospho-antibody Characterization

For in vitro cell line work, in-house developed extraction buffer AGLyse mainly containing high percentage of SDS and Tris with protease inhibitor and phosphatase inhibitor (Roche Applied Science) were used (23). Counterpart buffer AGLysewithout phosphatase inhibitor was prepared in order to allow AP to function at an expected level. Cells were harvested following 2 times of cold PBS washes and then followed by $4^{\circ} \mathrm{C}$ incubation with agitation for 30 mins. Supernatants were kept for downstream application. To generate paired AP treated samples, water adjusted $200 \mu \mathrm{g}$ of total protein lysate (per sample) was incubated with 10 $\mu$ g alkaline phosphatase (Sigma-Aldrich P0114-10KU) with $\mathrm{NaCl}(10 \mathrm{mM})$, $\mathrm{MgCl}_{2}(0.1 \mathrm{M})$, Tris $(5 \mathrm{mM})$ and Dithiothreitol DTT $(10 \mathrm{mM})$ at $37^{\circ} \mathrm{C}$ for 1 hour before RPPA processes. To generate a balanced AP reaction condition, protein lysates were adjusted to around $3 \mu \mathrm{g} / \mu \mathrm{l}$ with AGLyse and diluted with pure water to $2.2 \mu \mathrm{g} / \mu \mathrm{l}$ before reaction keeping all ratio of buffer components under the same condition. 
Tissue Sample Preparation and Lysis Condition Optimization for RPPA (FF and FFPE)

For fresh frozen sections ( $10 \mu \mathrm{m}$ thickness and ranging between $1 \mathrm{~cm}^{2}$ size across all samples), we compared our extraction method with CLB1 buffer (7M urea, 2M thiourea, 4\% CHAPS, 1\% Dithiotretol, $4 \mathrm{mM}$ spermidine, $2 \%$ pharmalyte, Roche protease inhibitor cocktail) under various conditions used previously that are compatible with Zeptosens detection system as well as other tissue protein extraction protocols for RPPA $(19,22,24)$. Generally, AGLyse buffer and CLB1 buffer were applied directly on tissues at approximately 50-100 $\mathrm{\mu l}$ and tissues were incubated for $30 \mathrm{mins}$ at room temperature or on ice and followed by centrifugation ( 5 mins at $15,000 \mathrm{~g}$ ). Supernatants were stored at $-80^{\circ} \mathrm{C}$ until downstream applications. The extraction processes were followed with or without extra sonication steps to facilitate the homogenization of tissues. For FFPE samples, we compared the standard CLB1 buffer with a range of lysing conditions (table 1a). We used the Qproteome FFPE protein extraction buffer accordingly to manufacturer instruction and literatures $(17,25)$. We also used a Tris-SDS based buffer $(20 \mathrm{mM}$ Tris-HCl,

$\mathrm{pH}=9$ with $2 \%$ SDS) optimized for nitrocellulose slide based RPPA previously with or without sonication in different combinations (26). For fresh frozen samples, CLB1 and AGLyse buffers (-AP) were used with additional intermediate processes and detailed optimization methods are summarized in table $1 \mathrm{~b}$.

Reverse Phase Protein Arrays

All extracted protein lysates and AP treated samples were measured for concentration using Bradford assay (Sigma-Aldrich) and diluted with array spotting buffer (CSBL1) to a final concentration of $0.2 \mu \mathrm{g} / \mu \mathrm{l}$ prior to array deposition. Zeptosens hydrophobic chips (ZeptoCHIP) were used (NMI-TT GmbH) and serial diluted samples (100\%, 75\%, 50\% and $25 \%$ ) prepared using a liquid handling robot (Tecan EVO75) were printed in duplicates using piezo-electric noncontact printer Nanoplotter NP2.0 (GeSiM). Chips were blocked with an ultrasonic nebulizer (ZeptoFOG blocking station) using Bovine Serum Albumin BSA containing BB1 buffer ( $3 \% \mathrm{w} / \mathrm{v})$ for 1 hour and followed by primary antibody incubation from various vendors overnight at $4^{\circ} \mathrm{C}$. Alexa-Fluor647-conjugated secondary antibodies (Abcam) were then incubated for 2 hours at $4^{\circ} \mathrm{C}$ prior to imaging with a ZeptoREADER instrument. All antibodies used for RPPA are listed in Supplemental S-2. All antibody incubations were carried out in CAB1 assay buffer (5\% BSA w/v). For on-chip protein loadings, duplicated slides were incubated with $0.45 \mu \mathrm{m}$ membrane-filtered freshlyprepared SyproRuby staining solutions (Invitrogen) for 30mins. Chips were then washed with ultra-pure water for 3 times prior to imaging on ZeptoREADER. To evaluate the non-specific signals potentially generated under buffer only conditions, buffer negative controls (buffer only) were prepared using the same procedure and spotted onto each array for parallel comparison. For experimental negative controls, spare arrays were incubated with secondary antibody only to assess non-specific binding and potential tissue derived autofluorescence.

Image Acquisition and Data Processing

Images acquired from ZeptoREADER were processed by ZeptoVIEW software. Three sequential images were acquired for each array at different exposure time between $0.5-10 \mathrm{~s}$. Under the $150 \mu \mathrm{m}$ spot diameter 
settings, all spots were automatically grid-aligned and the optimal image (without signal saturation) for each array was applied for quantification. The 8-point linear fitted median quantification values (relative fluorescence intensity RFI according to dilution curves) were generated by comparing with mean values of local positive reference standards (Alexa-Fluor647-conjugated BSA). RFIs were also local background corrected and negative controls (secondary antibody only) adjusted. RFI normalization was performed by dividing the background and negative control correctly values with the corresponding SyproRuby signals from individual spots generating the final data for subsequent analysis and statistical testing. For antibody quality evaluation, RNFI (relative normalized fluorescence intensity) was used to assess the signal-to-noise of individual spots.

Western Blotting

Extracted proteins were resolved in SDS-PAGE and transferred to PVDF membranes 1 hour at $4^{\circ} \mathrm{C}$ (Amersham Hybond). Membranes were blocked by 5\% BSA and hybridized with different primary antibodies at optimized dilutions indicated (Supplemental S-2). Chemiluminescent signals were captured by horse radish peroxidase (HRP) conjugated secondary antibodies for rabbit or mouse (SA000011/SA00001-2, Proteintech Groups) and visualized by enhanced chemiluminescence detection reagents (34578, ThermoFisher). The abundances of signal were digitally quantified by Image Lab software with a gel imaging system (ChemiDoc XRS+ Bio-Rad) and presented as arbitrary units of density. All antibody western blotting data are provided in Supplemental S-3.

Immunohistochemistry

Immunohistochemistry (IHC) was performed on formalin-fixed paraffin-embedded (FFPE) patient sections with an automated staining system (Dako Link48) using TTF1 primary antibodies (ZSGB-Bio Cat: ZM0270). After deparaffinization and rehydration in xylene and ethanol, antigen retrieval was performed in 1x EDTA retrieval solution (pH 8.0) (E-5134, Sigma) with heating. Inactivation of endogenous peroxidase was performed by adding enough drops of $3 \%$ hydrogen peroxide (H324-500, Fisher) to cover the whole section for $10 \mathrm{~min}$ and followed by primary antibody incubation at recommended working dilution. After 60 mins incubation at $37^{\circ} \mathrm{C}$, slides were washed twice with PBS, incubated with goat-anti-rabbit antibody (E046201, Dako) and then developed with DAB substrate (k3468, Dako) for 5-8 mins depending on the antibodies. Slides were counterstained with hematoxylin (CTS-1090, Biotechnologies) and were scanned with brightfield pathology microscope at 20X magnification (Aperio CS2, Leica) and processed by ImageScope software (Leica). Information on all other chemicals and reagents used in the article can be found in Supplemental S-2.

\section{Experimental Design and Statistical Rationale}

For all cell line-based phospho-antibody screening, three on-chip RPPA replicates were applied. For extraction method optimization in FFPE and FF, three independent experimental replicates were used for individual methods. For AP treatment in FF samples, three tissue replicates were used. For 36 phosphoantibody testing in FF samples, two tissue sections were used and each had two experimental replicates 
on parallel slides. For all melanoma and lung tissue FFPE samples, each patient had three parallel tissue sections treated as independent samples and processed for RPPA experiments.

To assess the consistency of the expression profiles under various extraction methods, pair-wise Pearson correlation was applied for both FFPE and FF samples. To gain an insight into the performance of phospho-antibodies in response to AP treatment in multiple cell lines, data obtained from above experiments were median-centered across samples and processed with unsupervised hierarchical clustering using Euclidean distance method. Log2 Fold-changes (LogFC: log ratio of RFI between AP treated and non-AP treated samples) were calculated for each antibody target (phosphor and nonphosphor controls) across all cell lines and ranked generating one factor to characterize the phosphoantibody performance. For all tissue sample replicate assessment, RFI and western blot comparison and expression correlation of two EGFR and p40 respectively, Pearson correlation was used. All inter-group comparisons were statistically analyzed by student's t-test, Mann-Whitney test or one-way ANOVA depending on the experimental contexts and $p$-value of 0.05 was considered significant unless elsewhere stated. To assess whether antibody specificities defined by AP-induced fold reduction can act as an independent factor to determine phospho-antibody quality, an antibody scoring system was used. The evaluation of "good" and "bad" antibodies was performed with an equal-weighted linear combination of spot quality, signal-to-noise ratio, dilution linearity, AP fold reduction multiplied by two binary factors assessed manually: positive reference quality and spot graininess/donut effects which are critical measures of the overall performance of phospho-antibodies and controls. The detailed formula calculation method and scoring description are presented in table 2. Based on this, we used different antibody scores (between 5-8) to define "good" and "bad" and their corresponding logFC to assess the predictive accuracies (AUC). We used an intermediate score considering the balance between AUC and the "good" and "bad" antibodies in real practice and performed Chi-square tests to evaluate the association between AP induced reduction (LogFC) and binary classified antibody categories ("good" or "bad"). All data analysis was carried out using R statistical and graphical interface.

\section{Results}

Phospho-antibody Profiling Using Alkaline Phosphatase Treatment in Cell Lines

A particular strength of RPPA is phosphoantibody profiling within cellular systems. RPPA enable the investigation of the phosphorylation status of many proteins in large numbers of samples. An important issue that has previously prevented the widespread adoption of RPPA for this purpose is the dependence on high antibody specificity and the establishment of suitable controls that are amenable to high throughput investigations. Alkaline phosphatase (AP) is an enzyme that indiscriminately removes phosphate from phosphorylated Ser, Thr or Tyr residues in proteins. We have previously developed a lysis buffer (AGLyse) that is highly efficient at lysing cells while at the same time providing an environment where AP retains its enzymatic activity. Here we show that AGLyse can be used in combination with a panel of commercially available cancer cell lines to directly assess the suitability of the phosphorylationspecific antibodies for the investigation of the perturbation of signal transduction pathways. 
A panel of 113 antibodies (106 anti-phospho-antibodies and seven protein-specific, but not phosphorylation-specific antibodies) was selected randomly from our antibody library and probed against eight cultured cell lysates that were treated or not treated with AP prior to printing on the RPPA. As expected, the removal of phosphates from the proteins in the cellular lysates resulted in a decrease of the binding of anti-phospho antibodies which was reflected in a clear separation of the observed relative fluorescence intensity (RFI) of untreated cellular lysates from that of AP-treated controls upon non supervised hierarchical clustering (Figure 1a). When the logFC was calculated for all 113 antibodies used for this experiment, followed by the sorting of antibodies by their response in 293T cells, the heatmap in Figure $1 \mathrm{~b}$ was obtained. All of the non-phosphorylation-specific antibodies did not show a strong response to AP treatment (as would be expected) and were therefore situated at the right end of the heatmap in Figure 1b together with phosphorylation-specific antibodies that did not show the expected response to AP-mediated protein dephosphorylation (antibodies such as pIGF1R $\left(\operatorname{Tyr}^{1135} / \mathrm{Tyr}^{1136}\right)$, pFAK $\left(\operatorname{Tyr}^{576}\right)$, pJAK $\left(\operatorname{Tyr}^{1022}\right)$, and pSmad3 $\left.\left(\operatorname{Thr}^{179}\right)\right)$. This effect was more pronounced, when the average logFC response was employed to sort the tested antibodies, resulting in a further shift of the nonphosphorylation specific antibodies to the right in the heatmap in Figure 1c.

We then asked whether the AP treatment could serve as an independent predictive factor to assess the suitability of anti-phosphorylation antibodies without considering other spot quality measures. Previously, we determined the suitability of antibodies for RPPA purposes using an antibody score that was calculated by taking into account six different factors: 1 . Spot quality score (percentage of the total sum of RFI excluding "poor" spots defined by ZeptoVIEW software); 2. Signal-to-noise ratio (the average fold difference between the RNFI of individual spots and the background obtained from individual spots); 3 . Dilution linearity score (averaged linearity generated by 8-point dilution across all samples); 4. Fold reduction score (average fold reduction in response to AP across all samples); 5 . Positive reference score (visual determination of the positive reference. This is a binary score: good 1 or bad 0 ); 6 . Spot graininess/donut effect (visual determination of homogenous staining and other signal related effects. This is a binary score: good 1 or bad 0 ). Factors 1 to 4 are equally weighted and categorized into three classes respectively (scored 1,2,3) with 3 having the best performance. Factors 5 and 6 are Boolean value that determine whether a particular antibody can be used or not. The sum of factors 1-4 multiplied by the two binary values from factors 5 and 6 gave rise to a ranked antibody score ranging from 0-12 (Supplemental Data S-4).

Defining all antibodies with an antibody score of 8 or higher (67 antibodies) as "Good" antibodies and antibodies with an antibody score of less than 8 (46 antibodies) as "Bad" antibodies permitted asking the question, if the AP-treatment based logFC value on its own could serve as a predictor of an antibody being "Good" or "Bad". The receiver operating characteristic curve (ROC curve) calculated for an antibodyscore cutoff value of 8 and a logFC cutoff value of -0.792 resulted in an area under the curve of 0.825 , indicating a reasonable ability of predicting the suitability of an anti-phosphorylation-specific antibody based on the AP-treatment dependent logFC value alone (Chi-square test $p<0.001$ ). To verify independently from the RPPA the veracity of results based on these antibodies, we selected 42 antibodies 
with $\log \mathrm{FC} \leq-0.792$ and performed Western Blots in all eight cell lines investigated here (Supplementary data S-2). 36 out of 42 antibodies (85\%) showed meaningful single bands at the expected sizes, confirming the suitability of this screening method for high-throughput approaches.

\section{Protein Extraction Optimization in FFPE and FF Tissue Specimen}

Following the establishment of the AP treatment-derived logFC value as a critical assay acceptance parameter in cell culture lysates, it was important to check the suitability of AGLyse based extraction for the analysis of clinically more relevant lysates of FFPE or FF tissue samples. For the analysis of breastderived FFPE samples, five different lysis methods were compared (Table 1a): 1. A commercial Qproteome FFPE sample extraction buffer; 2. A Tris/SDS based method that had previously been adopted for RPPA analyses $(15,18,21,26) ; 3$. The Tris/SDS based method (2) with an additional sonication step; 4. AGLyse extraction buffer procedure (including heating to $80^{\circ} \mathrm{C}$, sonication and AP inhibitors); 5 . AGLyse extraction buffer procedure (including heating to $80^{\circ} \mathrm{C}$, sonication without AP inhibitors)

A panel of 14 pre-validated antibodies (11 anti-phosphorylation antibodies and 3 total protein antibodies) was used to compare extraction methods 1-5. None of the extraction buffers produced a signal when it was applied to the array without any tissue sample. The correlation between the RPPA RFI signals generated from samples prepared using the 5 different extraction methods fluctuated but was acceptable for all antibodies (Figure 2A). The inter-method correlations for Methods 1-4 all showed correlation coefficients $R^{2}>0.9$ and a $p$-value of $p<0.01$ ) (Figure $2 b$ ). Interestingly, method 4 showed a higher correlation with the other three methods than with method 5 , despite the presence/absence of the AP inhibitors being the only difference between method 4 and method 5 . It is possible that either the AP inhibitor present in method 4 contributes to the solubilization of proteins from FFPE samples or that it inhibits intrinsic phosphatases in the FFPE lysates, a feature that is not necessary in the more denaturing methods 1-3.

For FF samples, the well characterized CLB1 lysis buffer was compared with Method 5 (Table 1b): 1 . CLB1 buffer extraction; 2. CLB1 buffer extraction with sonication and heating to $80^{\circ} \mathrm{C} ; 3$. CLB1 buffer extraction with heating to $80^{\circ} \mathrm{C}$. The same panel of 14 antibodies was used to compare extraction methods 5-8 on the FF tissue samples. Generally, using method 5, lower RFI signals were observed than for the extraction with CLB1 extraction buffer (Figure 3a). However, the correlation of the readout of the lysates prepared from FF tissue samples was very high for all 14 antibodies $\left(R^{2} \geq 0.93, p<0.01\right.$, Figure $\left.3 b\right)$. Therefore, we continued to apply alkaline phosphatase treatment with the established Method 5 (AGLyse without phosphatase inhibitor) in 3 parallel breast tissue selections and each with 3 independent replicates. Upon removal of the phosphatase inhibition, a signal reduction was observed for all 14 antibodies used in above experiments. Of the anti-phospho-antibodies, pP38 (Thr ${ }^{180} / \mathrm{Tyr}^{182}$ ), pHER2 $\left(\operatorname{Tyr}^{1221} / \operatorname{Tyr}^{122}\right)$ and pGSK3 $\beta\left(\right.$ Ser $\left.^{9}\right)$ and pERK1/2 $\left(\mathrm{Tyr}^{204}\right)$ showed a pronounced reduction of the signal intensity whereas negative controls (total antibodies against Akt, GSK-3 $\beta$ and a-tubulin) had a lesser reduction of the signal intensity in response to AP treatment (Figure 4). 
To assess the reproducibility of the method, we analyzed additional FF samples from two independent breast tissue sites each with two parallel extraction replicates using 36 anti-phosphorylation-antibodies validated in the previous screening experiment with AP treatment. A general reduction obtained from all phospho-proteins in response to AP treatment in FF tissues was observed (Figure 5a》 (paired t-test $p<0.05$ ). Site 2 displayed a lower RFI than site 1 (Figure $5 a$ and $5 b$ ), but the overall phosphorylation site occupation between site 1 and site 2 was very similar. Meanwhile, the overall expression exhibited no difference between two sites (Figure $5 b$ ). (non-paired t-test $p=0.11$ ) and high expression concordance was maintained significantly between experimental replicates (Figure 5c) (R2>0.9 and $p<0.001)$.

These data together provided initial evidence for the suitability of the AGLyse-based protein extraction method for FFPE and FF tissue specimen prior to RPPA profiling. It also demonstrates the feasibility of an AP treatment workflow for FF tissues indicating its potential for the validation of anti-phosphorylationantibodies in clinical tissue samples.

\section{Evaluation of RPPA Performance in Melanoma FFPE Samples}

As most clinically available samples are FFPE derived, we expanded our validation using a set of melanoma FFPE specimen. Three serially sectioned samples from a total of 63 patients were lysed and protein was extracted with AGLyse buffer. Extracted protein lysates were either analyzed using RPPA or using western blotting with a pre-validated Melan-A antibody, a specific melanocyte marker that is routinely used in histopathological diagnosis. Although extraction replicate No. 2 had relatively higher yields in comparison to other two replicates, the overall expression correlations between RPPA and western blotting were all retained at similar levels $\left(R^{2}=0.44-0.53, p<0.05\right)$ (Figure 6a and Supplemental Figure S2).

Due to the semi-quantitative nature of western blotting as well as its sub-optimal reproducibility for interexperimental quantification, we also equally divided western blotting data into three categories based on their signal intensities ( 0 , low and high) and plotted against RPPA data. Two out of three extraction replicates showed significantly different expression at least between low and high groups (ANOVA $p<0.05$ ) indicating largely the consistency between these two methods (Figure 6b). We then interrogated further with 4 additional antibodies validated for RPPA (GAPDH, LAG3, PD-L1 and S100) and performed pair-wise comparison between replicates and for all targets tested, strong correlations were observed $\left(\mathrm{R}^{2}\right.$ ranging from 0.54 to $0.97, p<0.05$ ) (Figure 7 ). Taken together, by assessing a cohort of 63 melanoma patient samples, we further warranted the robustness of the established extraction methodology with FFPE samples and proved their compatibility for downstream RPPA analysis strengthening its application potential in clinical settings.

\section{RPPA Characterization of Lung Cancer FFPE Tissues}

Performing RPPA based analysis of FFPE-tissue protein extracts generated using the AGLyse buffer was further evaluated through the analysis of 3 subtypes of lung cancers versus normal lung tissue samples. This analysis was performed using a panel of 13 histopathological protein expression biomarkers some 
of which are routinely used for lung cancer subtyping. 20 Non-Small Cell Lung Cancer (NSCLC) patients, of which 10 were adenocarcinoma (ADC) and 10 were squamous cell carcinoma (SCC) and 10 small cell lung cancer (SCLC) patients were analyzed. 10 adjacent non-cancerous lung epithelium samples were included as control. Of these patients a total of 120 FFPE slides were acquired comprising 3 serial sections per patient for protein extraction and subsequential RPPA profiling. Protein quantification showed similar extraction efficiency between parallel sectioned samples despite one set (rep2) having significantly lower protein yields than others $(p<0.05)$ (Supplemental Figure S3). For RPPA profiling, adenocarcinoma markers Napsin A, cytokeratin 7, TTF1, squamous cell carcinoma markers p40, p63 and small cell lung cancer markers TTF1 were used. Additionally, EGFR, VEGFR3, VEGF, PD-1, topoisomerase, tubulin- $\beta I I I$ and ROS1 were also used to obtain in-depth protein expression profiles on the same sample sets. Unsupervised hierarchical clustering revealed distinct expression patterns of proteins in the three lung cancer subtypes separating them from non-cancerous epithelium. This was supported by displaying the score plot of a PCA analysis that indicated a partial separation not only of non cancerous tissue extracts from cancer tissue extracts, but also a partial separation of the three lung cancer subtypes from each other (Figure 8a/b). Interestingly, the results confirmed what is known about the biology of the different lung cancer subtypes. This can be more clearly appreciated when the tissue sample sets are grouped according to their clinical subtypes. ADC tissue samples showed high expression levels of Ck7 and Napsin A and reduced protein expression of p63 and p40. SCC tissue samples in contrast showed the opposite pattern with minimal protein expression levels of CK7 and Napsin A and comparatively higher levels of p40 and p63. Both subtypes, ADC and SCC had EGFR expression levels that were significantly higher than those observed in SCLC extracts and in control tissue. SCLS extract displayed higher protein expression levels than the three other tissue extracts for TTF1, which was also reflected at IHC level (Figure 10 This protein had the lowest expression level in the SCC tissue samples, as expected). Of interest, as for other markers, all of them developed a similar increasing pattern with baseline expression in normal lung tissues, slight up-regulation in ADC and further elevated level in SCC and highest expression in SCLC suggesting a potential expression signature associated with the aggressiveness of cancer and probable for SCLC molecular stratification (Figure 9). For EGFR and p40, as two antibody strains were available for RPPA respectively, both were tested against each other and our RPPA data showed optimal consistency for both targets $\left(R^{2}=0.94, p<0.001\right)$ again reconfirming the technical robustness and compatibility for FFPE derived tissue specimen (Supplemental Figure S4).

\section{Discussion}

The application of novel high-plex proteomic technologies for clinical tissue sample profiling has been significantly impeded by stringent sample pre-processing, preservation and other technical related factors. Although numerous efforts have been made to develop clinical-ready methodologies, such analytical tools are yet not widely available. As an immunology based targeted proteomic approach, RPPA has advantages for the quantitative assessment of clinical tissue specimens due to its simple preparation workflow, minimal sample consumption, high sensitivity and parallel high-plexing capacity (27). Therefore, many others have made early breakthroughs in optimizing pre-analytical variables in

Page 12/28 
order to obtain reliable data for clinical interpretation especially on FFPE samples $(13,15,16,18,19,21$, 25,26 ). We developed a new protein extraction workflow by employing an in-house buffer (AGLyse) and comprehensively tested its reliability on both sectioned FF and FFPE tissue materials alongside with other extraction conditions used in previous literatures.

Different from other buffer compositions, AGLyse has a milder denaturing characteristic allowing alkaline phosphatase to function to certain extent and thus it has great potential for phospho-antibody screening which is critical for downstream RPPA application. This method was preliminarily used in previous experiments where the specificity of pP53 $\left(\mathrm{Ser}^{15}\right)$ antibody was tested (23). By non-discriminative stripping of all phosphor-groups on protein analytes, 106 randomly selected phospho-antibodies were evaluated along with 7 antibodies targeting total proteins for controls as they were expected with minimal reduction in expression. For all lysates generated from 8 cell lines, a general reduction in the presence of AP was apparent as post-AP treated samples perfectly clustered together. By color-annotating the antibodies (phosphor in yellow and total in black) in the ranked fold-change heatmap (FC ranked by 293T and the rest cell lines were aligned accordingly), the performance of individual phospho-antibody can be easily determined and as anticipated, all antibodies targeting total proteins were enriched in the right regions indicating the reliability of the method. Notably, a portion of phospho-antibodies had even elevated expression upon AP induction probably due to antibody specific reasons either via interaction with alkaline phosphatase non-specifically or intrinsic incompatibility with RPPA workflow and therefore were classified as unsuitable antibodies for RPPA application The on-slide antibody performance is another analytical factor in RPPA antibody evaluation especially for fluorescence-based detection approaches and therefore multiple parameters are routinely used mainly by checking signal-tobackground ration, spot quality and reproducibility, dilution linearity, positive reference spot quality and signal saturation $(8,28,29)$. These are sometimes tedious and require manual interpretation causing unavoidable errors. We sought to evaluate whether the cell line-based fold reduction could serve as an independent deterministic parameter regardless of others. Under a LogFC $=-0.792$ and antibody score $=8$ (AUC $=0.825)$, Chi-square tests revealed a strong association between multi-parameter evaluation and LogFC alone $(p<0.001)$ providing initial evidence for antibody quality control without considering other routine decisive parameters, however this approach certainly needs validation with expansion of antibodies. As we termed this approach as a bottom-up phospho-antibody screening method, western blots were performed with antibodies categorized with LogFC $\leq-0.792$ following RPPA screening. Of the 42 phospho-antibodies tested, 36 have successfully showed single dominant bands by probing multiple cell lines whereas 6 had non-specific detection in contrast stressing the need of WB validation for RPPA. Together our approach for high throughput phospho-antibody evaluation for RPPA has proven to be workable, however, screen-verified antibodies still require thorough western blotting confirmation to determine the target size and specificity as non-specific reduction catalyzed by AP could also cause affinity binding on any other de-phosphorated proteins. Nevertheless, this approach is extremely efficient for phospho-antibodies pre-screening and adaptable for RPPA profiling. 
Upon initial exploration, our attention had moved toward the assay compatibility with tissue specimen. Two types of tissue (FFPE and FF) were assessed in parallel and various tissue protein extraction protocols were also used as reference. They served as positive controls during our experimental procedure and allowed side-by-side comparison between different extraction methods. For FFPE samples, commercial Qproteome extraction (Method 1) and Tris-SDS based extraction (Method 2/3) both generated decent signals over the buffer only background across 14 selected antibodies (phosphor and total). Though certain degrees of variation on some targets were observed (GSK3 $\beta$, $\alpha$-tubulin, Akt and pPI3K-p85(Tyr $\left.{ }^{467 / 199}\right)$ in particular) with or without sonication in Method 2, their overall correlation was acceptable. This could be partially attributed to the inter-slide variation of tissues or alteration of posttranslational modification prior to fixation and paraffin embedding. AGLyse based methods (Method 4/5) both produced comparative signals with slight reduction of some phosphorylated proteins (Method 5), which was probably caused by removal of phosphatase inhibition. Despite these facts, their overall concordance with other pre-established methods was strikingly high $\left(R^{2}\right.$ between $\left.0.75-0.96, p<0.05\right)$ suggesting the reliability of the methodology for FFPE derived sample profiling. As for FF samples, previously characterized CLB1 buffer was used in various extraction conditions (Method 6-8) as positive controls $(21,22,30)$. All of them have generated analyzable signals over the buffer background with heatinduced methods (Method 7/8) having highest signal levels throughout on the same set of antibodies used in FFPE sample characterization. This suggested heat-induction may also be critical in FF tissue protein extraction to untangle protein molecules. AGLyse mediated extraction (Method 5) had again generated usable signals above the buffer only background (Method 5 buffer only), despite their global lower signals compared to other CLB1 based methods. Apart from that, the inter-method correlation was remarkably high ( $R^{2}$ 0.93-0.96) indicating FF samples may be more stable in general for RPPA phosphoproteomic profiling. Of note, under both extraction conditions (FFPE and FF), tissue autofluorescence was negligible suggesting the feasibility of the data. By incorporating AP treatment, we further evaluated the performance of those 14 phospho-antibodies used in above experiments on FF samples and allof the phospho-antibodies exhibited expected reduction upon AP treatment whereas total antibodies (GSK3 $\beta$, atubulin and Akt) remained unaffected. Data from the inter-extraction replicates were reproducible suggesting the consistency of the method and notable variation on some targets (pS6 $\left(\mathrm{Ser}^{235 / 236}\right)$ and $\mathrm{pp} 44 / 42\left(\mathrm{Tyr}^{204}\right)$ ) could be explained by intra-patient heterogeneity. Extended analysis on 36 pre-validated phospho-antibodies also proved the robustness and fidelity of AGLyse with AP treatment for phosphoantibody screening in FF tissues. Antibodies used for RPPA profiling need thorough validation across sample types and especially for samples derived from tissue origins, high complexity may result in crossreactivities leading to misinterpretation of data $(8,25,31)$. Our AGLyse buffer based extraction and treatment workflow hinted on a novel approach for fast phospho-antibody characterization compatible for both in-vitro cultured cells and FF tissues and may serve as an efficient tool in RPPA and related applications. As phosphorylation is the most extensively studies post-translational modification in cancer biology and plays crucial roles throughout tumor progression, our method may vastly accelerate phospho-antibody validation efficacy for tissue proteomics in clinical translational research, however this 
is currently restricted to sample denaturing conditions and other applications such as immunohistochemistry and immunofluorescence must to be tested independently.

Preliminary success on FFPE samples have led us to focus on their applicability in larger cohorts of clinical specimen. On a batch of 63 patient FFPE samples (3 serial sections for each patient with a total of 189 slides), our protein extraction method generated similar levels of protein yields across three replicates and cross comparison of Melan-A expression (a melanoma cytosolic marker) between RPPA and western blotting suggested a decent correlation between those two detection methods $\left(R^{2}\right.$ between $0.44-0.53 p<0.001)$. By equally grouping western blotting data into three subsets based on Melan-A expression (nil, median and high), there was an increasing tendency of RPPA signals accordingly with western blotting groups for all three experimental replicates regardless of the majority showing statistically non-significance ( $p>0.05)$. As either western blotting or IHC are semi-quantitative and digitally transformed quantitative readouts from western blotting are extremely difficult for interexperimental comparison, direct assessment of RPPA data with conventional western blotting and IHC might be implausible and many others have shown moderate side-by-side comparison results $(16,17,19$, 32). RPPA in that sense as a bulk sample high-plex analytical approach may serve as an assistive clinical diagnostic tool due to its incapability of resolving heterogeneities that have significant impact on expression profiles. Despite this technical downside, RPPA has great potentials for multiplexed proteomic analysis and this is reflected by measuring multiple markers on a single tissue section. Inter-replicate expression of PDL1, LAG3, S100B and GAPDH provided fairly consistent data $\left(R^{2}\right.$ between $0.52-0.97, p<$ $0.05)$ and general expression patterns can be easily obtained.

In a final validation experiment, our purpose was to evaluate whether RPPA can be utilized for cancer subtype classification. Same extraction and RPPA quantification workflow were employed on a set of 40 lung FFPE tissues wherein 30 belonged to three major clinical subtypes (ADC, SCC and SCLC) and 10 adjacent normal lung epithelial samples were used as controls. Protein concentrations between three replicates again revealed general consistency across individual extractions. Using lung cancer pathological differentiation markers (Napsin A, cytokeratin7, TTF1, p40/p63 and EGFR) together with other validated antibodies (VEGFR3, VEGF, ROS1, topoisomerase, Tubulin $\beta$-III and PD1), both unsupervised heatmap and PCA analysis distinguished individual subtypes successfully and SCLC exhibited most pronounced dissimilarity compared to normal lung tissues and NSCLC. Though two NSCLC subtypes were closely correlated, clustering still separated those two subtypes expectedly. Data presented in semi-supervised clustering and box plots of individual subtypes also demonstrated their concordance with clinical pathological features. These were shown by up-regulation of EGFR in ADC and SCC, down-regulation of TTF1 in SCC but increased in ADC and SCLC and up-regulation of Napsin A and Ck7 in ADC (all ANOVA p < 0.05). Though p40 (a SCC differentiation marker) was up-regulated in SCC and $A D C$ in part as compared to normal lung (ANOVA $p<0.05$ ), it failed to discriminate SCC and ADC ( $p>$ 0.05) and oppositely, although p63 (a SCC differentiation marker) was able to distinguish SCC and ADC, it failed to separate from normal epithelium. p40 was discovered as a more sensitive marker for SCC subtyping than p63, however in RPPA profiling its performance was inferior to the latter one (33). Despite 
intrinsic variability amongst all patients tested, this could partially be attributed to the defects of RPPA in dealing heterogeneous samples and the antibody compatibility for quantification in high precision especially for FFPE derived resources. Of interest, all other protein markers tested (ROS1, PD-1, VEGFR3, VEGF, topoisomerase and tubulin $\beta$-III) constituted an elevated expression signature with normal lung tissues having the lowest expression, NSCLC showing intermediate expression and the neuroendocrine subtype SCLC presenting the highest levels. This was in agreement with the aggressiveness of the clinical phenotypes and prevalent expression pattern of topoisomerase, tubulin $\beta-I I I, V E G F, ~ V E G F R 3$ and PD-1 in SCLC were previously documented at histopathological or cellular levels (34-38). ROS1 rearrangement was extensively characterized in lung cancer and our finding is largely in line with the findings previously in NSCLC and SCLC, both of which had elevated expression (39). Given that the C-terminal kinase domain function was likely retained (antibody targeting C-terminal of ROS1) and small cell transformation results in loss of fusion or mutation in kinase domains conferring resistance to tyrosine kinase inhibitors (TKIs), our data indicate yet a functional competent ROS1 throughout tumor progression and strengthens the need to disentangle ill-defined mechanisms of truncated ROS1 isoforms during tumor transformation (40). Though our ROS1 antibody was not designed specifically for fusion detection in IHC, RPPA has eminent potentials to serve as a fast and efficient pre-screening tool using mutant specific antibodies prior to IHC and fluorescence in-situ hybridization (FISH) (41).

\section{Conclusions}

Conclusively, by utilizing an in-house developed buffer system, our systematic work provides a novel RPPA extraction method that is universally compatible for fast phospho-antibody screening in cell lines and FF tissues. We also conducted rigorous validation for its robustness in analyzing FFPE derived samples on melanoma and lung cancer patient cohorts. Albeit phosphoprotein quantification is challenging in FFPE due to pre-analytical factors, total protein can be semi-quantitatively profiled with RPPA for clinical phenotype stratification. Data derived from lung cancer patients are pathologically meaningful and suggest a potential signature associated with oncogenesis and progression. With indispensable validation of antibodies across multiple tissues, RPPA may serve as a biomarker profiling tool for clinical archived samples as well as a for pre-clinical and clinical screening and companion diagnosis under specific contexts.

\section{List Of Abbreviations}

$A D C$, adenocarcinoma; $A P$, alkaline phosphatase; $A U C$, area under curve; $B S A$, bovine serum albumin; CAB1, cell assay buffer1; CK7, cytokeratin7; CLIA, clinical laboratory improvement amendments; CLB1, cell lysis buffer1; CSBL1, spotting buffer for lysates; EGF, epidermal growth factor; EGFR, epidermal growth factor receptor; ER estrogen receptor; FC, fold-change; FCS, fetal calf serum; FF, fresh frozen, FFPE, formalin-fixed and paraffin-embedded; FISH, fluorescence in-situ hybridization; HER, epidermal growth factor receptor; IGF, Insulin growth factor; IHC, immunohistochemistry; NSCLC, non-small cell lung cancer; PD-1, programmed cell death protein-1; PR, progesterone receptor; PTM, post-translational modification; 
$\mathrm{RFI}$, relative fluorescence intensity; RNFI, relative normalized fluorescence intensity; RPPA, reverse phase protein arrays; SCC, squamous cell carcinoma; SCLC, small cell lung cancer; SDS, Sodium Dodecyl Sulfate; TKI, tyrosine kinase inhibitor; TTF1, Thyriod transcription factor-1; VEGF, Vascular Endothelial Growth Factor; VEGFR, Vascular Endothelial Growth Factor Receptor; WB, western blots.

\section{Declarations}

\section{Ethics approval and consent to participate}

The study included tumor specimens from all patients who underwent surgical resection (in.Beijing Cancer Hospital). All tissue samples had informed consent from the patients as documents in Beijing Cancer Hospital for research purpose.

\section{Consent for publication}

Not applicable

\section{Availability of data and materials}

The datasets used and/or analysed during the current study are available from the corresponding author on reasonable request (nan.wang@fynnbio.com). These include and not limited to original RPPA data.

\section{Completing interests}

Fynn Biotechnologies holds commercial interests on AGLyse protein extraction buffer and has an associated patent (ZL 20171 1323182.6) and offers RPPA screening as a commercial service Dr Achim Treumann has acted in the past as a consultant for Fynn Biotechnologies.

\section{Funding}

This work was fully supported by Fynn Biotechnologies Research \& Development funding sources. Those include study design, external sample collection (tissues, cell lines, transient expression cells), exterimentation, data analysis and interpretation and manuscript writing.

\section{Author contributions}

Nan Wang: Conceptualization, Methodology, Formal analysis, Writing-Original Draft, Investigation, Resources, Project administration; Li Zhang: Methodology, Validation, Investigation, Resources, WritingReview \& Editing; Qi Ying: Software, Validation, Formal analysis, Investigation, Data curation; Zhentao Song: Software, Formal analysis, Data curation, Visualization; Aiping Lu: Resources, Supervision; Achim Treumann: Conceptualization, Methodology, Writing-Review \& Editing; Zhaojian Liu: Resources, Supervision; Tao Sun: Resources, Supervision, Writing-Review \& Editing, Zhiyong Ding: Methodology, Writing-Review \& Editing, Resources, Supervision 


\section{Acknowledgments}

We thank VigeneBio for providing 293T transient over-expression clones as western blotting controls. We thank Yuping Deng and Wenping Yu for assisting externally with all collaborators to complete the study. We also thank Song from Beijing Cancer Hospital for assistive work in providing pathological resources.

\section{Footnotes:}

Dr Achim Treumann has moved from Newcastle University since Mar 2019 and is now Director of Analytical Formulation Sciences and Site Head at KBI Biopharma BV, Belgium.

\section{References}

1. Masuda M, Yamada T. Signaling pathway profiling using reverse-phase protein array and its clinical applications. Expert Rev Proteomics. 2017;14(7):607-15.

2. Winters $M$, Dabir B, Yu M, Kohn EC. Constitution and quantity of lysis buffer alters outcome of reverse phase protein microarrays. Proteomics. 2007;7(22):4066-8.

3. Hennessy BT, Lu Y, Gonzalez-Angulo AM, Carey MS, Myhre S, Ju Z, et al. A Technical Assessment of the Utility of Reverse Phase Protein Arrays for the Study of the Functional Proteome in Nonmicrodissected Human Breast Cancers. Clin Proteomics. 2010;6(4):129-51.

4. Ambroz KL, Zhang Y, Schutz-Geschwender A, Olive DM. Blocking and detection chemistries affect antibody performance on reverse phase protein arrays. Proteomics. 2008;8(12):2379-83.

5. Tibes R, Qiu Y, Lu Y, Hennessy B, Andreeff M, Mills GB, et al. Reverse phase protein array: validation of a novel proteomic technology and utility for analysis of primary leukemia specimens and hematopoietic stem cells. Mol Cancer Ther. 2006;5(10):2512-21.

6. Spurrier B, Ramalingam S, Nishizuka S. Reverse-phase protein lysate microarrays for cell signaling analysis. Nat Protoc. 2008;3(11):1796-808.

7. Tegnebratt T, Ruge E, Bader S, Ishii N, Aida S, Yoshimura Y, et al. Evaluation of efficacy of a new MEK inhibitor, RO4987655, in human tumor xenografts by [(18)F] FDG-PET imaging combined with proteomic approaches. EJNMMI Res. 2014;4(1):34.

8. Akbani R, Becker KF, Carragher N, Goldstein T, de Koning L, Korf U, et al. Realizing the promise of reverse phase protein arrays for clinical, translational, and basic research: a workshop report: the RPPA (Reverse Phase Protein Array) society. Mol Cell Proteomics. 2014;13(7):1625-43.

9. van Oostrum J, Calonder C, Rechsteiner D, Ehrat M, Mestan J, Fabbro D, et al. Tracing pathway activities with kinase inhibitors and reverse phase protein arrays. Proteomics Clin Appl. 2009;3(4):412-22.

10. Espina V, Mueller C, Liotta LA. Phosphoprotein stability in clinical tissue and its relevance for reverse phase protein microarray technology. Methods Mol Biol. 2011;785:23-43. 
11. Guo H, Gao M, Lu Y, Liang J, Lorenzi PL, Bai S, et al. Coordinate phosphorylation of multiple residues on single AKT1 and AKT2 molecules. Oncogene. 2014;33(26):3463-72.

12. Sheehan KM, Calvert VS, Kay EW, Lu Y, Fishman D, Espina V, et al. Use of reverse phase protein microarrays and reference standard development for molecular network analysis of metastatic ovarian carcinoma. Mol Cell Proteomics. 2005;4(4):346-55.

13. Berg D, Hipp S, Malinowsky K, Bollner C, Becker KF. Molecular profiling of signalling pathways in formalin-fixed and paraffin-embedded cancer tissues. Eur J Cancer. 2010;46(1):47-55.

14. Malinowsky K, Raychaudhuri M, Buchner T, Thulke S, Wolff C, Hofler H, et al. Common protein biomarkers assessed by reverse phase protein arrays show considerable intratumoral heterogeneity in breast cancer tissues. PLoS One. 2012;7(7):e40285.

15. Berg D, Wolff C, Malinowsky K, Tran K, Walch A, Bronger H, et al. Profiling signalling pathways in formalin-fixed and paraffin-embedded breast cancer tissues reveals cross-talk between EGFR, HER2, HER3 and uPAR. J Cell Physiol. 2012;227(1):204-12.

16. Negm OH, Muftah AA, Aleskandarany MA, Hamed MR, Ahmad DA, Nolan CC, et al. Clinical utility of reverse phase protein array for molecular classification of breast cancer. Breast Cancer Res Treat. 2016;155(1):25-35.

17. Wulfkuhle JD, Berg D, Wolff C, Langer R, Tran K, Illi J, et al. Molecular analysis of HER2 signaling in human breast cancer by functional protein pathway activation mapping. Clin Cancer Res. 2012;18(23):6426-35.

18. Becker KF, Schott C, Hipp S, Metzger V, Porschewski P, Beck R, et al. Quantitative protein analysis from formalin-fixed tissues: implications for translational clinical research and nanoscale molecular diagnosis. J Pathol. 2007;211(3):370-8.

19. Berg D, Langer R, Tran K, Walch A, Schuster T, Bronger H, et al. Protein microarray-based comparison of HER2, estrogen receptor, and progesterone receptor status in core biopsies and surgical specimens from FFPE breast cancer tissues. Appl Immunohistochem Mol Morphol. 2011;19(4):300-5.

20. Assadi M, Lamerz J, Jarutat T, Farfsing A, Paul H, Gierke B, et al. Multiple protein analysis of formalin-fixed and paraffin-embedded tissue samples with reverse phase protein arrays. Mol Cell Proteomics. 2013;12(9):2615-22.

21. Bader S, Zajac M, Friess T, Ruge E, Rieder N, Gierke B, et al. Evaluation of Protein Profiles From Treated Xenograft Tumor Models Identifies an Antibody Panel for Formalin-fixed and Paraffinembedded (FFPE) Tissue Analysis by Reverse Phase Protein Arrays (RPPA). Mol Cell Proteomics. 2015;14(10):2775-85.

22. Gromov P, Celis JE, Gromova I, Rank F, Timmermans-Wielenga V, Moreira JM. A single lysis solution for the analysis of tissue samples by different proteomic technologies. Mol Oncol. 2008;2(4):36879.

23. Wu CE, Esfandiari A, Ho YH, Wang N, Mahdi AK, Aptullahoglu E, et al. Targeting negative regulation of p53 by MDM2 and WIP1 as a therapeutic strategy in cutaneous melanoma. $\mathrm{Br} \mathrm{J}$ Cancer. 2018;118(4):495-508. 
24. Pirnia F, Pawlak M, Thallinger GG, Gierke B, Templin MF, Kappeler A, et al. Novel functional profiling approach combining reverse phase protein microarrays and human 3-D ex vivo tissue cultures: expression of apoptosis-related proteins in human colon cancer. Proteomics. 2009;9(13):3535-48.

25. Schuster C, Malinowsky K, Liebmann S, Berg D, Wolff C, Tran K, et al. Antibody validation by combining immunohistochemistry and protein extraction from formalin-fixed paraffin-embedded tissues. Histopathology. 2012;60(6B):E37-50.

26. Guo H, Liu W, Ju Z, Tamboli P, Jonasch E, Mills GB, et al. An efficient procedure for protein extraction from formalin-fixed, paraffin-embedded tissues for reverse phase protein arrays. Proteome Sci. 2012;10(1):56.

27. Boellner S, Becker KF. Reverse Phase Protein Arrays-Quantitative Assessment of Multiple Biomarkers in Biopsies for Clinical Use. Microarrays (Basel). 2015;4(2):98-114.

28. Voshol H, Ehrat M, Traenkle J, Bertrand E, van Oostrum J. Antibody-based proteomics: analysis of signaling networks using reverse protein arrays. FEBS J. 2009;276(23):6871-9.

29. Pawlak M, Schick E, Bopp MA, Schneider MJ, Oroszlan P, Ehrat M. Zeptosens' protein microarrays: a novel high performance microarray platform for low abundance protein analysis. Proteomics. 2002;2(4):383-93.

30. Wang DC, Wang X. Systems heterogeneity: An integrative way to understand cancer heterogeneity. Semin Cell Dev Biol. 2017;64:1-4.

31. Weissenstein U, Schneider MJ, Pawlak M, Cicenas J, Eppenberger-Castori S, Oroszlan P, et al. Protein chip based miniaturized assay for the simultaneous quantitative monitoring of cancer biomarkers in tissue extracts. Proteomics. 2006;6(5):1427-36.

32. Roncador G, Engel P, Maestre L, Anderson AP, Cordell JL, Cragg MS, et al. The European antibody network's practical guide to finding and validating suitable antibodies for research. MAbs. 2016;8(1):27-36.

33. Suzuki M, Muroi A, Nojima M, Numata A, Takasaki H, Sakai R, et al. Utility of a Reverse Phase Protein Array to Evaluate Multiple Biomarkers in Diffuse Large B-Cell Lymphoma. Proteomics Clin Appl. 2020;14(1):e1900091.

34. Bishop JA, Teruya-Feldstein J, Westra WH, Pelosi G, Travis WD, Rekhtman N. p40 (DeltaNp63) is superior to $\mathrm{p} 63$ for the diagnosis of pulmonary squamous cell carcinoma. Mod Pathol. 2012;25(3):405-15.

35. Lv C, Liu X, Zheng Q, Chen H, Yang X, Zhong J, et al. Analysis of topoisomerase I expression and identification of predictive markers for efficacy of topotecan chemotherapy in small cell lung cancer. Thorac Cancer. 2018;9(9):1166-73.

36. Powell S, Kaizer A, Koopmeiners JS, Iwamoto C, Klein M. High expression of class III beta-tubulin in small cell lung carcinoma. Oncol Lett. 2014;7(2):405-10.

37. Schultheis AM, Scheel AH, Ozretic L, George J, Thomas RK, Hagemann T, et al. PD-L1 expression in small cell neuroendocrine carcinomas. Eur J Cancer. 2015;51(3):421-6. 
38. Chen P, Zhu J, Liu DY, Li HY, Xu N, Hou M. Over-expression of survivin and VEGF in small-cell lung cancer may predict the poorer prognosis. Med Oncol. 2014;31(1):775.

39. Tanno S, Ohsaki Y, Nakanishi K, Toyoshima E, Kikuchi K. Human small cell lung cancer cells express functional VEGF receptors, VEGFR-2 and VEGFR-3. Lung Cancer. 2004;46(1):11-9.

40. Uguen A, De Braekeleer M. ROS1 fusions in cancer: a review. Future Oncol. 2016;12(16):1911-28.

41. Lin JJ, Langenbucher A, Gupta P, Yoda S, Fetter IJ, Rooney M, et al. Small cell transformation of ROS1 fusion-positive lung cancer resistant to ROS1 inhibition. NPJ Precis Oncol. 2020;4:21.

42. Viola P, Maurya M, Croud J, Gazdova J, Suleman N, Lim E, et al. A Validation Study for the Use of ROS1 Immunohistochemical Staining in Screening for ROS1 Translocations in Lung Cancer. J Thorac Oncol. 2016;11(7):1029-39.

\section{Tables}

Due to technical limitations, Table1a, Table1b and Table2 are only available as a download in the Supplemental Files section.

\section{Figures}

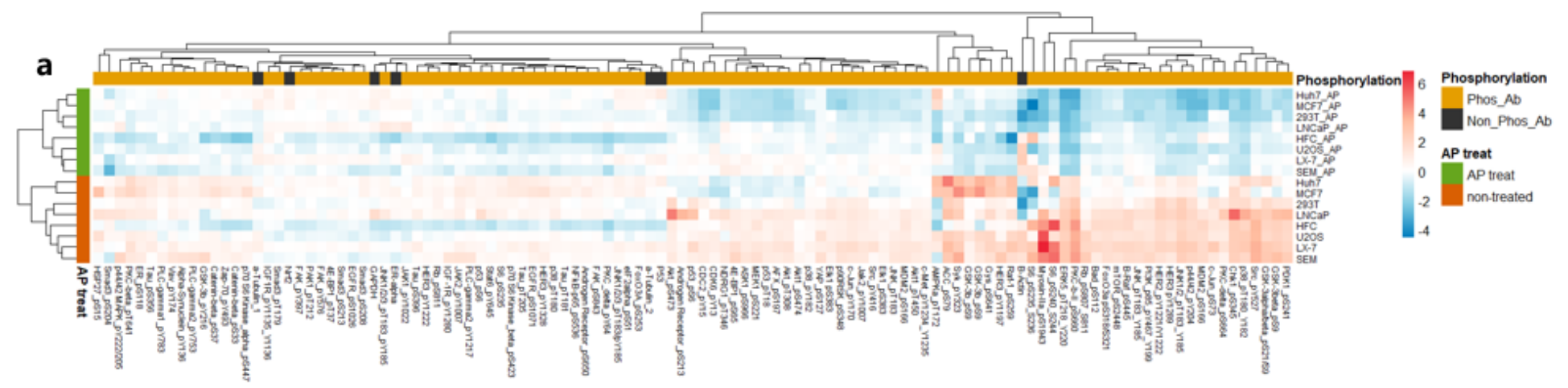

b

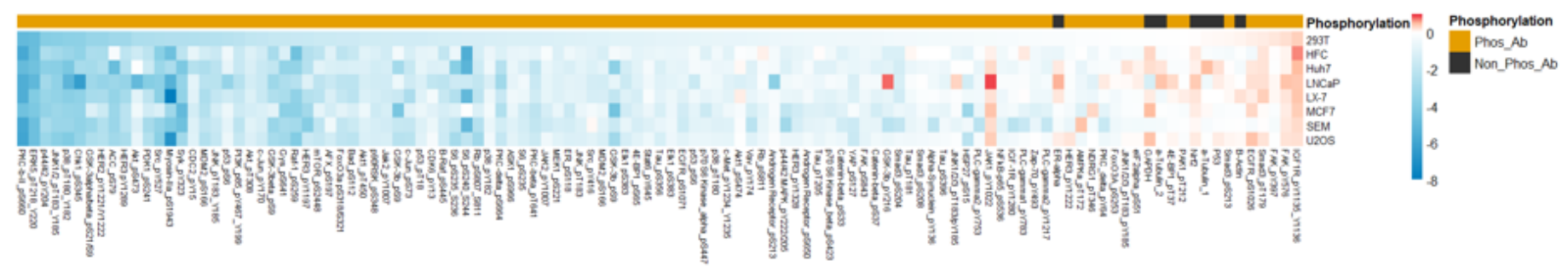

c

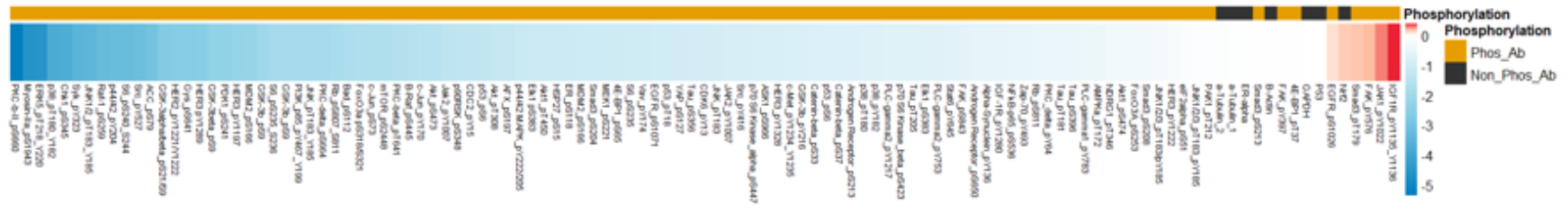




\section{Figure 1}

Assessment of alkaline phosphatase treatment induced effect across 8 cell lines versus non-treated controls. a. Unsupervised hierarchical clustering of lysates generated with AGLyse with or withour additional AP treatment across 8 cell lines (SEM, LX-7, U2OS, HFC, LNCaP, 293T, MCF7 and Huh7). Phospho-antibodies are marked in yellow and total antibodies (Non-phosphor) are marked in black. b. LogFC reduction level of individual antibodies across cell lines. Individual target LogFC is ranked by 293T cells from the lowest to highest (left to right) and rest of cell lines are matched according to LogFC positions of individual targets. c. Averaged logFC across 8 cell lines and data are ranked from lowest to highest (left to right).

a

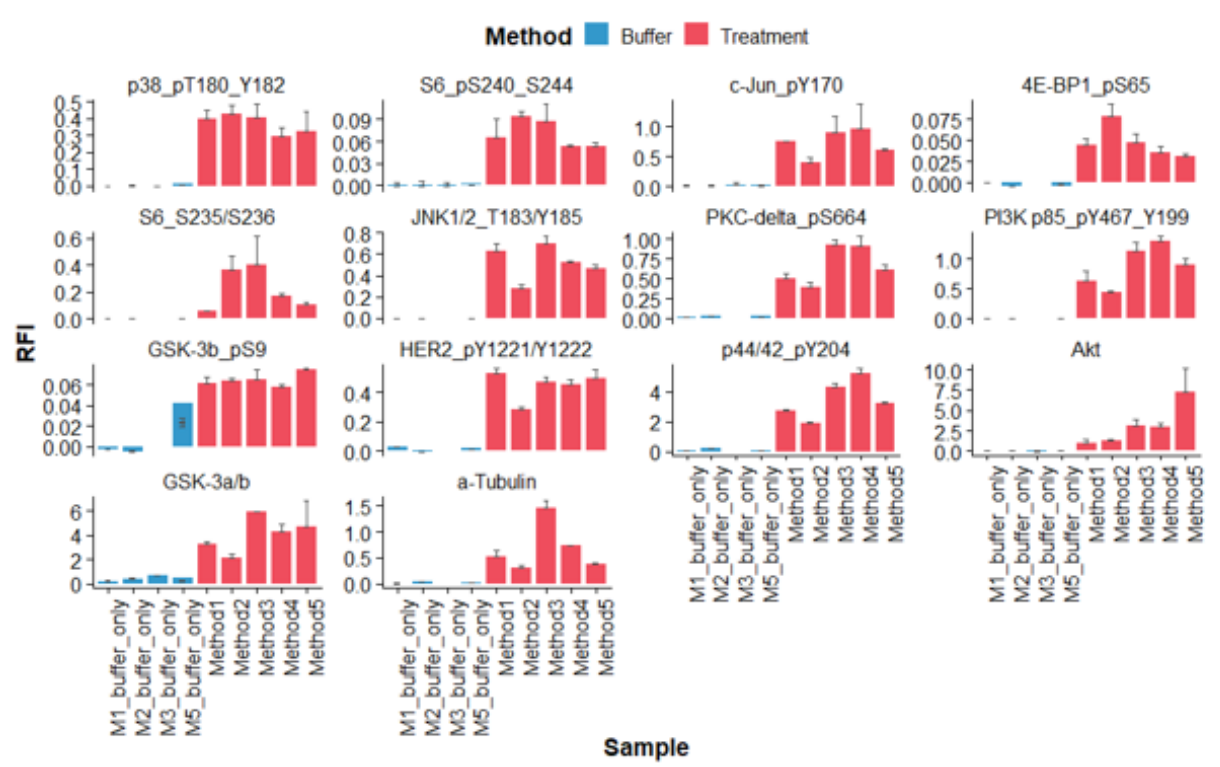

b

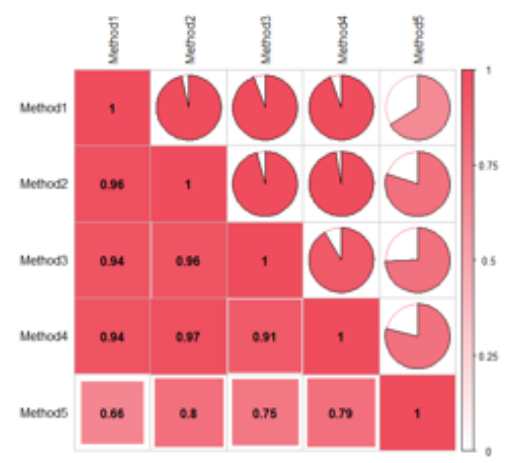

\section{Figure 2}

RPPA profiling of multiple targets using 4 extraction methods on FFPE samples. a. Expression profiling of 4 extraction methods (method 1-4) presented by arbitrary units (relative fluorescent intensity RFI) and error bars represent standard deviation from 3 independent extractions. Method 5 is under the same condition as Method 4 without alkaline phosphatase inhibitor. Blue bars are on-chip buffer only controls assessed within the same experiments. b. Inter-method correlation based on 14 protein markers and R2 are shown from white to red (0-1). 
a

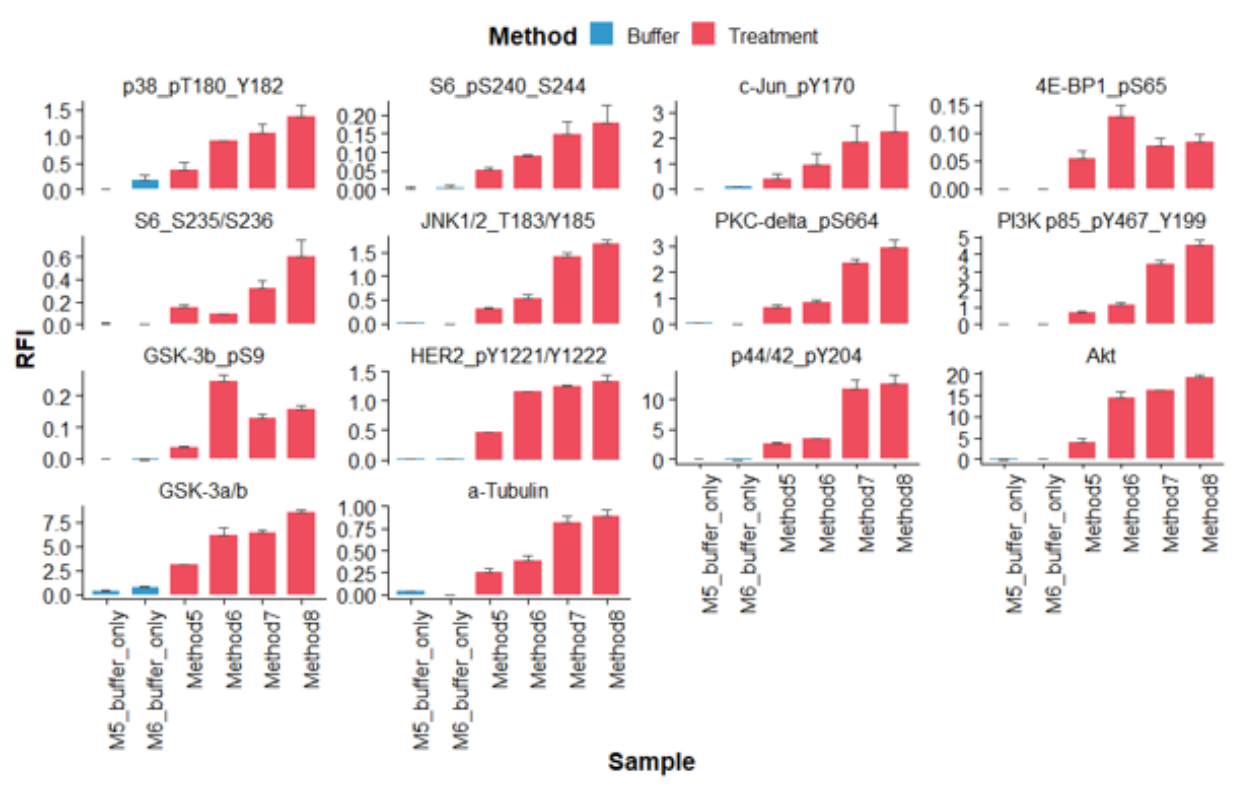

b

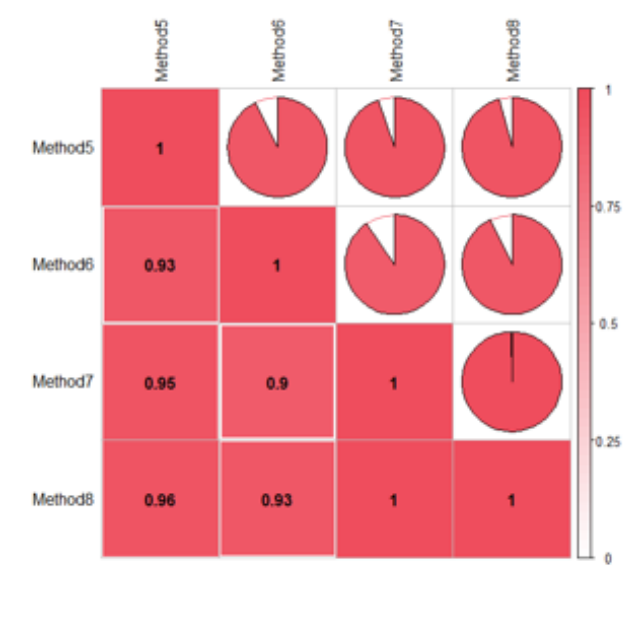

\section{Figure 3}

RPPA profiling of multiple targets using 4 extraction methods on FF samples. a. Expression profiling of 3 extraction methods (method 5-8) presented by arbitrary units (relative fluorescent intensity RFI) and error bars represent standard deviation from 3 independent extractions. Blue bars are on-chip buffer only controls assessed within the same experiments. b. Inter-method correlation based on 14 protein markers and R2 are shown from white to red (0-1).

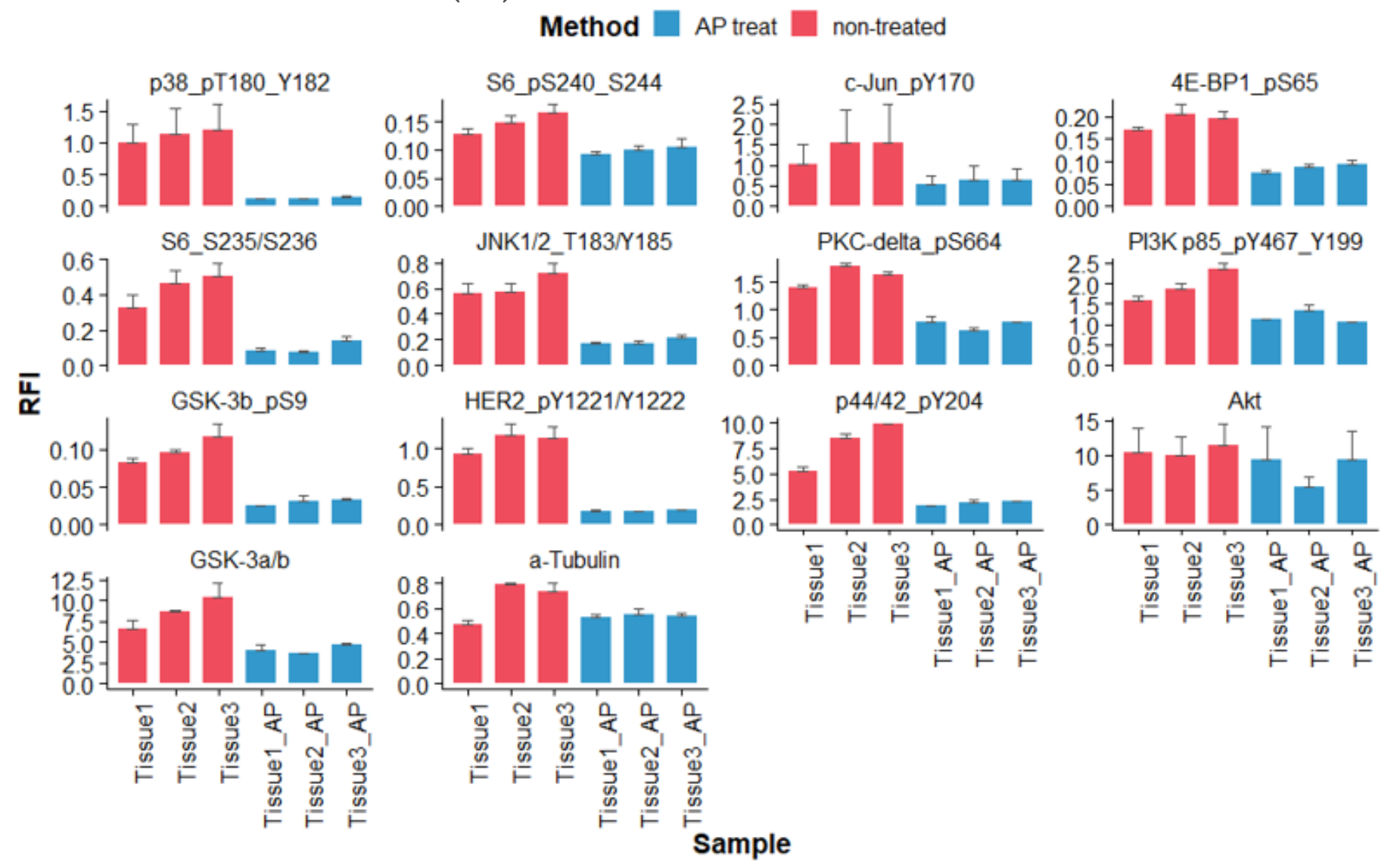




\section{Figure 4}

Comparison of AP-treated and non-treated FF samples using RPPA profiling on independent replicates Non-treated controls are depicted in red and AP-treated are in blue. Each bar represents 3 individual extractions from parallel slides derived from same tissue origins. Error bars are standard deviation between 3 experimental replicates.
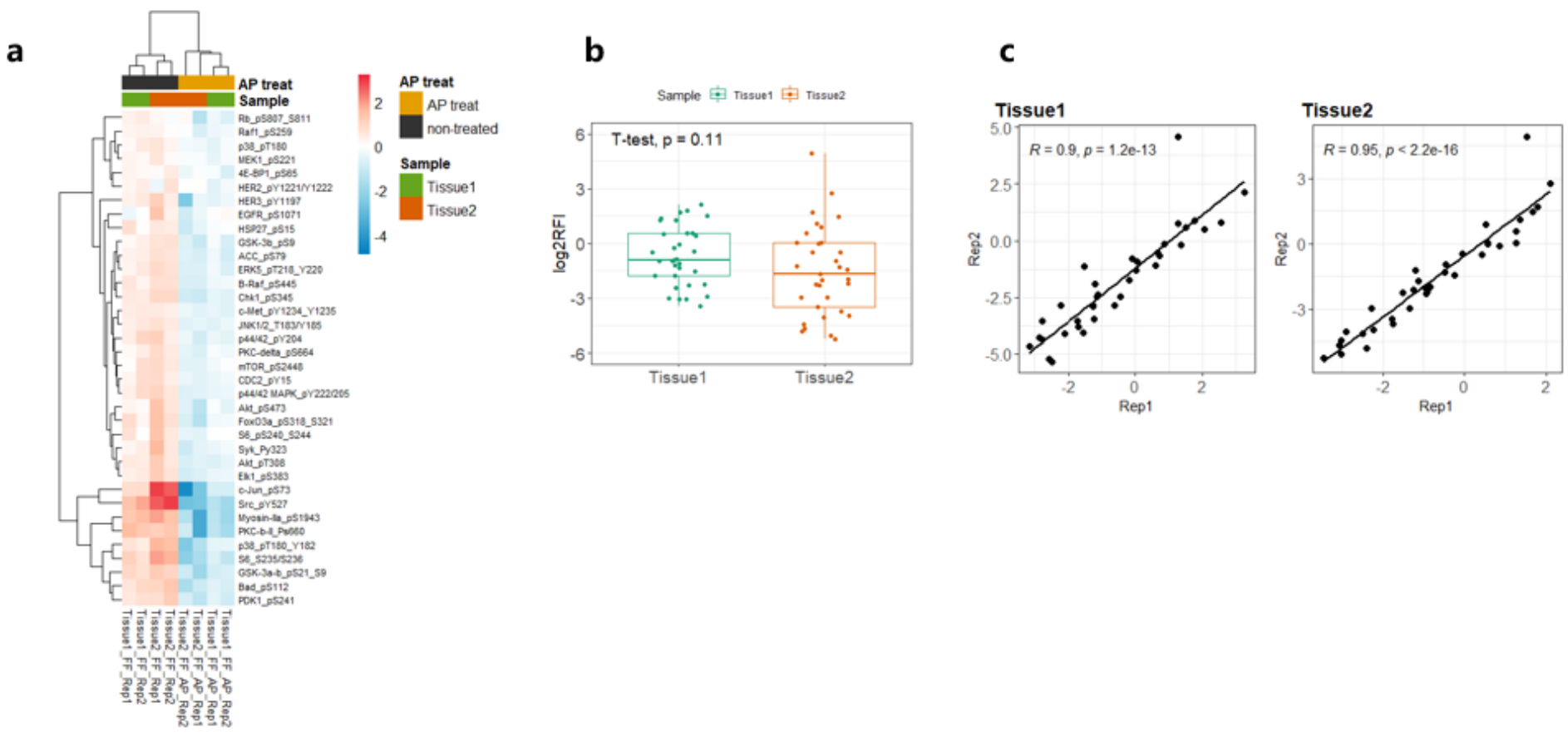

\section{Figure 5}

RPPA assessment of AP treatment in FF tissue samples. a. Unsupervised hierarchical clustering of tissue samples treated with (yellow) or without AP (black) based on RPPA profiling of 36 phospho-markers. Rep1 and Rep2 represent two independent tissue sites derived from the same patient and for each site, extraction was performed on two parallel sections separately. b. Overall expression profiling between two tissue sites (Rep1 and Rep2). Data are shown in log2 transformed RFI (Relative fluorescence intensity) and unpaired t-test was used to assess the differential expression between two tissue sites. c. Interreplicate expression correlation presented by RFI from individual tissue sites. Pearson correlation coefficients are shown. 
a

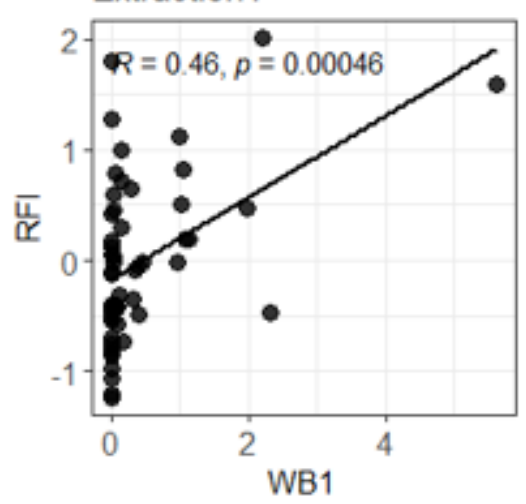

b

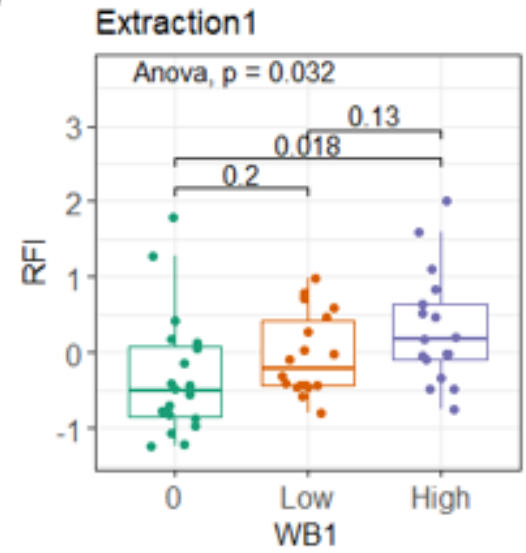

Extraction2

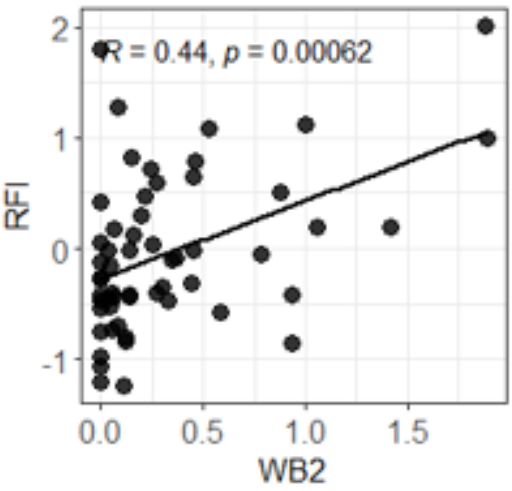

WB1 审 0 审 Low

\section{Extraction2}

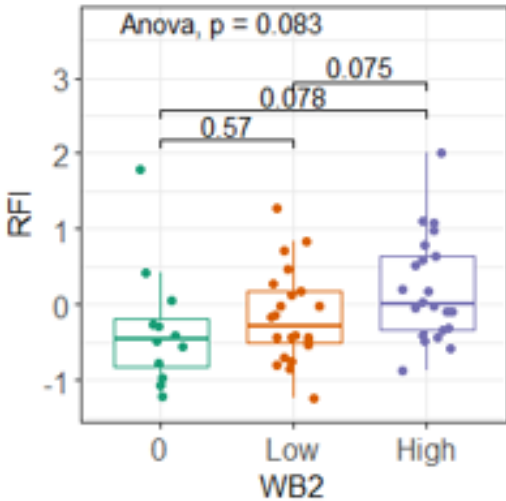

\section{Extraction3}

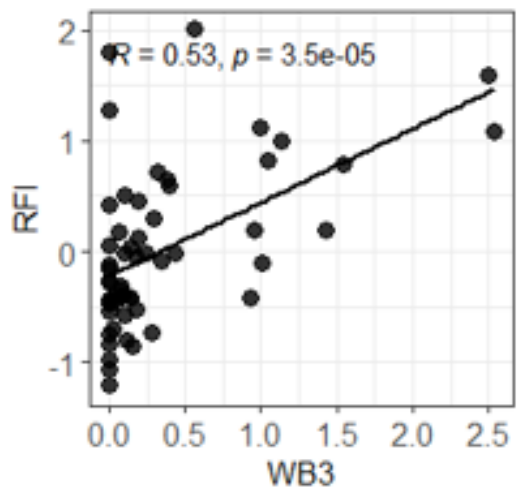

Extraction 3

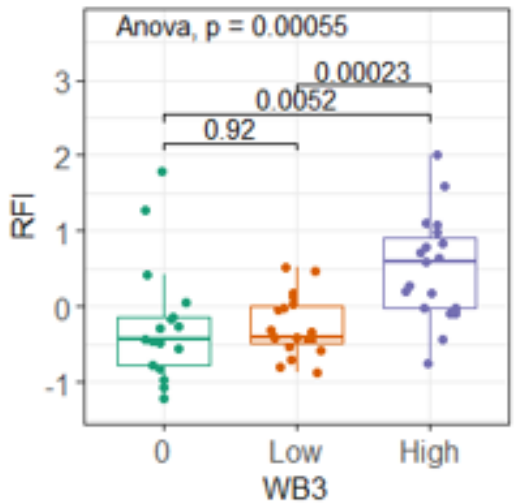

Figure 6

Expression correlation between RPPA and western blot in melanoma 63 FFPE patient samples. a. Direct plotting of expression levels between western blot (arbitrary unit) and RPPA (RFI) from three independent experimental replicates. R2 and Pearson correlation are shown accordingly. b. Boxplots representing RFI from three equally divided expression categories based on western blot data. Statistical test results are shown (ANOVA). 
GAPDH
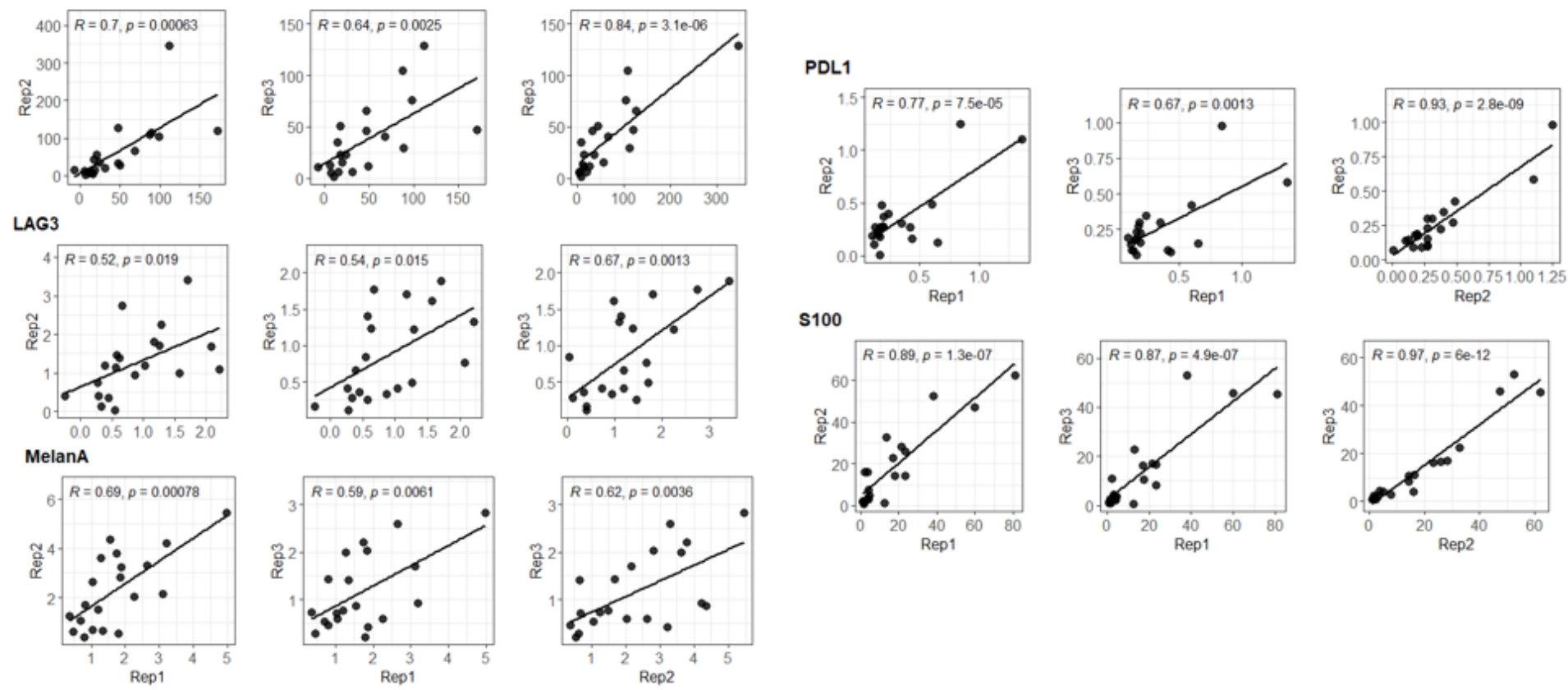

Figure 7

RPPA expression correlation between individual experimental replicates. Five protein markers (GAPDH, PDL1, LAG3, S100, MelanA) in 63 melanoma FFPE patients were tested and R2 and Pearson correlation coefficients are shown respectively.

a

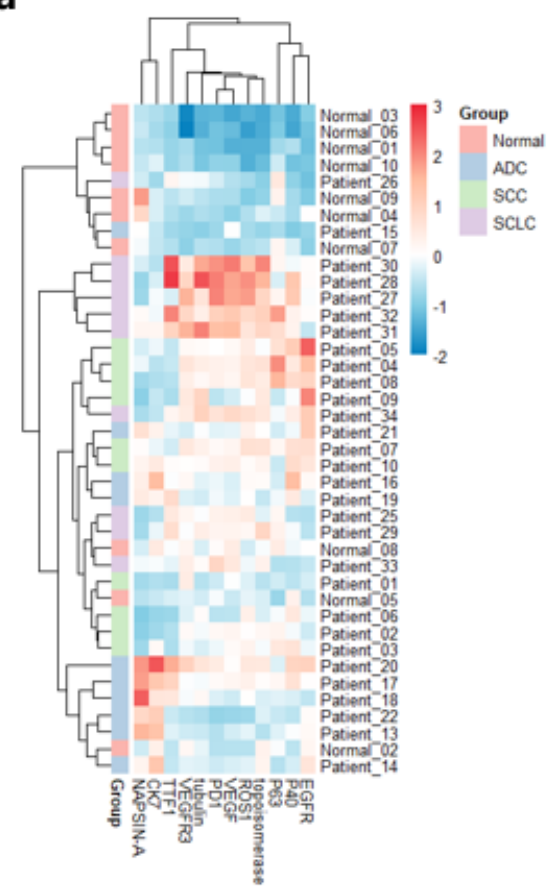

b

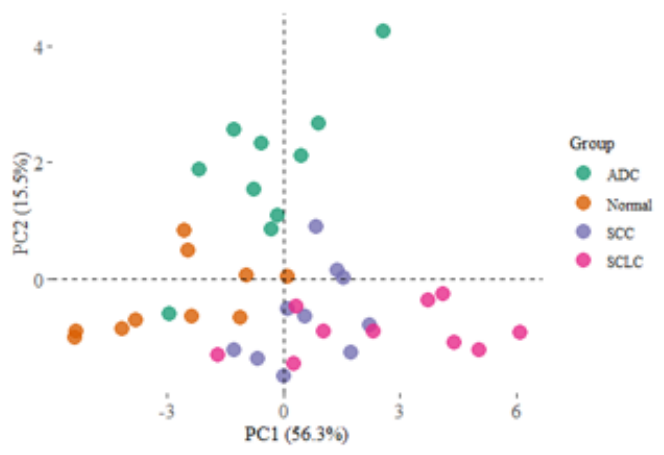

c

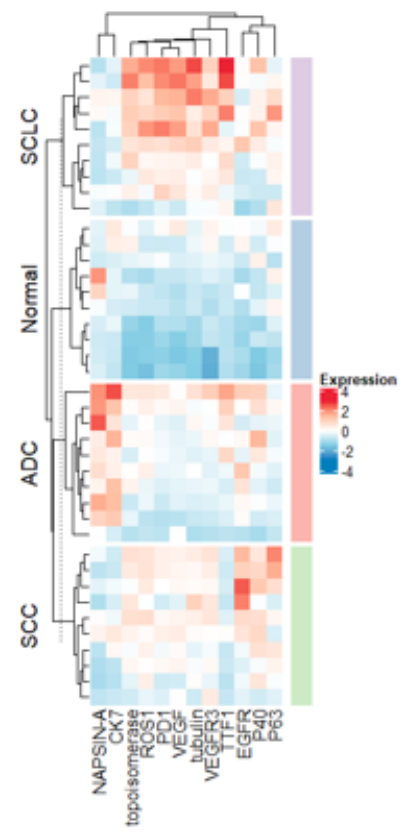

Figure 8 
Clustering analysis based on 12-protein based RPPA profiling of lung cancer patient subtypes. a. Unsupervised hierarchical clustering of three lung cancer subtypes (adenocarcinoma ADC, squamous cell carcinoma SCC, small cell lung cancer SCLC) and para-tumor FFPE samples. b. PCA plot of three lung cancer subtypes and normal lung tissues and only PC1 and PC2 are presented.
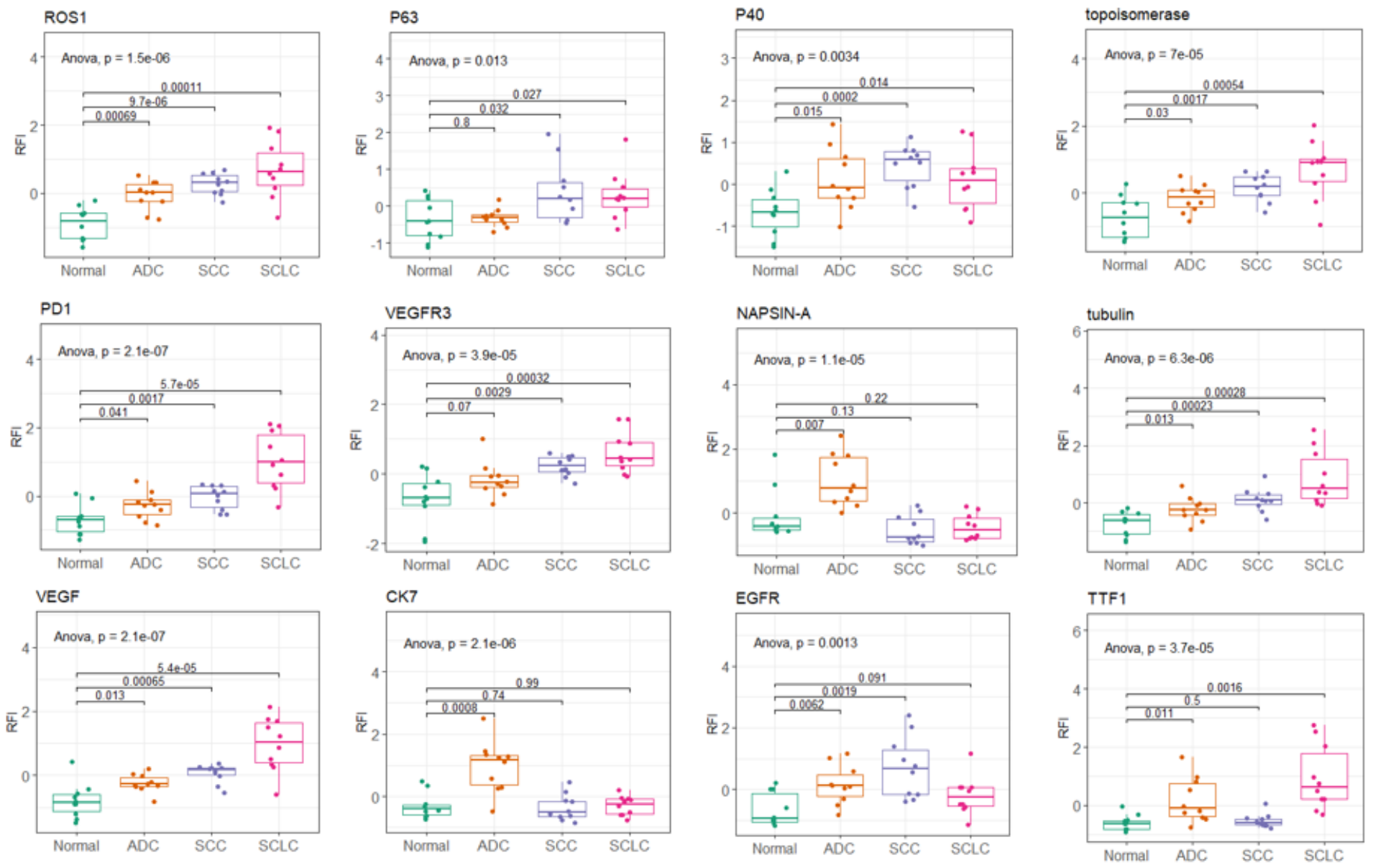

\section{Figure 9}

Boxplots of individual protein expression shown by RFI in three lung cancer subtypes. Adenocarcinoma ADC, squamous cell carcinoma SCC, small cell lung cancer SCLC and para-tumor FFPE samples are compared and statistical test results are shown (ANOVA).

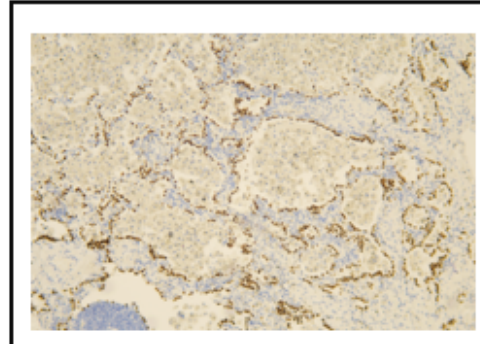

Normal lung

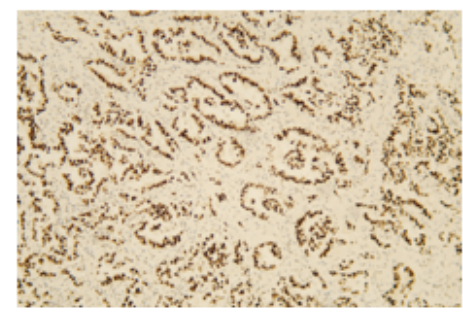

$A D C$

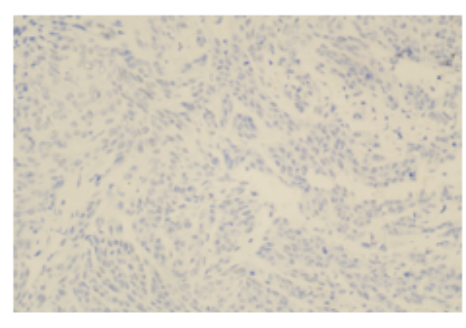

SCC

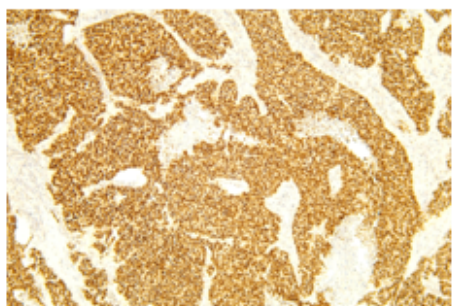

SCLC 
Immunohistochemistry staining of TTF1 across three lung cancer subtypes and adjacent normal tissues. Images were taken at $100 \mathrm{X}$ resolution.

\section{Supplementary Files}

This is a list of supplementary files associated with this preprint. Click to download.

- Tables.pdf

- SupFigures.pptx

- Supplementary1celllineinfo.xlsx

- Supplementary2antibodyandreagentinformation.xlsx

- Supplementary3antibodyWBdata.pptx

- Supplementary4antibodyscoring.xlsx 